\title{
Exclusive photoproduction of quarkonium in proton-nucleus collisions at energies available at the CERN Large Hadron Collider
}

\author{
G. Sampaio dos Santos and M. V. T. Machado \\ High Energy Physics Phenomenology Group, GFPAE IF-UFRGS Caixa Postal 15051, CEP 91501-970, Porto Alegre, RS, Brazil
}

(Received 3 December 2013; published 3 February 2014)

\begin{abstract}
In this work we investigate the coherent photoproduction of $\psi(1 S), \psi(2 S)$, and $\Upsilon$ states in the proton-nucleus collisions in the CERN Large Hadron Collider (LHC) energies. Predictions for the rapidity distributions are presented using the color dipole formalism and including saturation effects that are expected to be relevant at high energies. Calculations are done at the energy 5.02 $\mathrm{TeV}$ and also for the next $\mathrm{LHC}$ run at $8.8 \mathrm{TeV}$ in proton-lead mode. Discussion is performed on the main theoretical uncertainties associated to the calculations.
\end{abstract}

DOI: 10.1103/PhysRevC.89.025201

PACS number(s): 12.38.Bx, 13.60.Hb, 24.85.+p

\section{INTRODUCTION}

The exclusive quarkonium photoproduction has being investigated both experimentally and theoretically in recent years as it allows to test perturbative quantum chromodynamics. The masses of these heavy mesons, $m_{V}$, give a perturbative scale for the problem even in the photoproduction limit, $Q^{2} \rightarrow 0$. An important feature of these exclusive processes at the high energy regime is the possibility to investigate the hard pQCD Pomeron exchange. For this energy domain hadrons and photons can be considered as color dipoles in the mixed light cone representation, where their transverse size can be considered frozen during the interaction [1]. Therefore, the scattering process is characterized by the color dipole cross section describing the interaction of those color dipoles with the nucleon or nucleus target. Such an approach is intuitive and allows to introduce information on dynamics beyond the leading logarithmic QCD approach. The information of the meson formation is given by their wave functions and to compute predictions for their excited states is a reasonably easy task [2].

In the present work, we investigate the exclusive production of $J / \psi$, its radially excited $\psi(2 S)$ state, and $\Upsilon(1 S)$ in protonnucleus collisions in the CERN Large Hadron Collider (LHC) energy range. The theoretical framework considered is the light-cone dipole formalism [1], where the $Q \bar{Q}$ fluctuation (color dipole) of the incoming quasireal photon (from the protons or nuclei) interacts with the target via the dipole cross section and the result is projected in the wave function of the observed hadron. At high energies, the transition of the regime described by the linear dynamics of emissions chain to a new regime where the physical process of recombination of partons becomes important is expected. It is characterized by the limitation on the maximum phase-space parton density that can be reached in the hadron wave function, the so-called parton saturation phenomenon (see reviews in Ref. [3]). The transition is set by saturation scale $Q_{\text {sat }} \propto x^{\lambda}$, which is enhanced in the nuclear case. We will make use of this formalism to evaluate the corresponding cross sections. In the $p A$ collisions considered here, it is possible to investigate at the same time the dynamics on the photon-proton cross section and on the photon-nucleus cross section. The dominant contribution comes from the photon-proton interaction as the photon flux due to the nucleus is higher compared to that due to the proton. It will be shown that the photon-nucleus contribution is relevant at large rapidities and increasingly important for heavy quarkonia as the $\Upsilon$ states. The paper is organized as follows. In the next section we summarize the main theoretical information to compute the rapidity distribution of quarkonia in $p A$ collisions. In Sec. III we present the numerical calculations and discuss the main theoretical uncertainties and a comparison with another approaches is done. In the last section we show the main conclusions.

\section{THEORETICAL FRAMEWORK AND MAIN EXPRESSIONS}

Let us consider the proton-nucleus interaction at large impact parameter $\left(b>R_{p}+R_{A}\right)$ and at ultrarelativistic energies. In this regime we expect the electromagnetic interaction to be dominant. In this case, the cross section of quarkonium $V$ production can be evaluated within the Weizsäcker-Williams approximation as a product of the photon flux emitted by one of the colliding participants and the cross section of quarkonium photoproduction on the remaining hadron or nucleus. In particular, in proton-lead collisions if the quarkonium rapidity, $y$, is positive in the nucleus beam direction its rapidity distribution reads as [4]

$$
\begin{aligned}
\frac{d \sigma}{d y}(\mathrm{~Pb}+p \rightarrow \mathrm{Pb}+p+V)= & \frac{d N_{\gamma}^{\mathrm{Pb}}(y)}{d \omega} \sigma_{\gamma p \rightarrow V+p}(y) \\
& +\frac{d N_{\gamma}^{p}(-y)}{d \omega} \sigma_{\gamma \mathrm{Pb} \rightarrow V+\mathrm{Pb}}(-y),
\end{aligned}
$$

where $\frac{d N_{\gamma}(y)}{d \omega}$ is the corresponding photon flux and $y=$ $\ln \left(2 \omega / m_{V}\right)$, with $\omega$ being the photon energy. The case for the inverse beam direction is straightforward. We use the Weiszäcker-Williams method to calculate the flux of photons from a charge $Z$ nucleus [4]:

$$
\frac{d N_{\gamma}^{\mathrm{Pb}}}{d \omega}=\frac{2 Z^{2} \alpha_{e m}}{\pi}\left[\xi K_{0}(\xi) K_{1}(\xi)+\frac{\xi^{2}}{2}\left(K_{1}^{2}(\xi)-K_{0}^{2}(\xi)\right)\right],
$$

where $\xi=\omega\left(R_{p}+R_{A}\right) / \gamma_{L}$ and $\gamma_{L}$ is the Lorentz boost of a single beam. For the proton case, we use the following 
expression for the photon energy spectrum [5]:

$$
\begin{aligned}
\frac{d N_{\gamma}^{p}}{d \omega}= & \frac{\alpha_{e m}}{2 \pi}\left[1+\left(1-\frac{2 \omega}{\sqrt{s}}\right)^{2}\right] \\
& \times\left(\ln \chi-\frac{11}{6}+\frac{3}{\chi}-\frac{3}{2 \chi^{2}}+\frac{1}{3 \chi^{3}}\right),
\end{aligned}
$$

where $\chi=1+\left(Q_{0}^{2} / Q_{\min }^{2}\right)$ with $Q_{0}^{2}=0.71 \mathrm{GeV}^{2}$ and $Q_{\min }^{2}=$ $\omega^{2} / \gamma_{L}^{2}$.

In the present analysis, we consider the photonhadron/nucleus scattering in the color dipole formalism, in which most of the energy is carried by the hadron and the photon dissociates into a quark-antiquark pair long before the scattering. Such an approach turns out the analysis of the small- $x$ dynamics of the hadron wave function clearer and more intuitively. The probing projectile fluctuates into a quark-antiquark pair with transverse separation $r$ long after the interaction, which then scatters off the target (proton or nucleus) [1]. Accordingly, the cross section for exclusive photoproduction of quarkonium off a nucleon target is given by [2]

$$
\sigma_{\gamma p \rightarrow V p}=\frac{1}{16 \pi B_{V}}\left|\sum_{h, \bar{h}} \int d z d^{2} r \Psi_{h, \bar{h}}^{\gamma} \sigma_{\mathrm{dip}}(x, r) \Psi_{h, \bar{h}}^{V *}\right|^{2},
$$

where $\Psi^{\gamma}$ and $\Psi^{V}$ are the light-cone wave function of the photon and of the vector meson, respectively. The Bjorken variable is denoted by $x$, the dipole cross section by $\sigma_{\text {dip }}(x, r)$, and the diffractive slope parameter by $B_{V}$. Here, we consider the energy dependence of the slope using a Regge motivated expression [6]. In our numerical calculation, corrections for the skewedness and real part of the amplitude have also been included [7]. On the other hand, the exclusive photoproduction off nuclei for coherent processes can be computed in a simple way in the large coherence length $\ell_{c} \gg R_{A}$ limit $[8,9]$ :

$$
\begin{aligned}
\sigma_{\gamma A \rightarrow V A}= & \int d^{2} b|S(x, b ; A)|^{2}, \\
S(x, b ; A)= & \sum_{h, \bar{h}} \int d z d^{2} r \Psi_{h, \bar{h}}^{\gamma}(z, r) \Psi_{h, \bar{h}}^{V *}\left(z, r, m_{V}\right) \\
& \times\left[1-\exp \left(-\frac{1}{2} R_{G} \sigma_{\operatorname{dip}}(x, r) T_{A}(b)\right)\right],
\end{aligned}
$$

where $T_{A}(b)=\int d z \rho_{A}(b, z)$ is the nuclear thickness function. In the numerical evaluations, we have considered the boosted Gaussian wave function [10] and the phenomenological saturation model proposed in Ref. [11] which encodes the main properties of the saturation approaches. We call attention to the fact that the parameters of the model [11] have been updated with the recent high-precision combined data from HERA in Ref. [12]. Large $x$ effects have been introduced by multiplying the dipole cross section by a factor $(1-x)^{\alpha}$. The nuclear ratio for the gluon density is denoted by $R_{G}\left(x, Q^{2}, b\right)$, which will be discussed in the following section.

\section{RESULTS AND DISCUSSIONS}

Before presenting the predictions for the quarkonia production let us discuss the role played by the gluon shadowing in the input cross section $\sigma_{\gamma A \rightarrow V A}$. It was recently shown that the color dipole approach with $R_{G}=1$ is not able to describe the ALICE data for coherent production of $J / \psi$ at $2.76 \mathrm{GeV}$ $[13,14]$. The midrapidity cross section is overestimated by a factor of two. The reason is that for $R_{G}=1$ in the photon-nucleus cross section the nuclear effect included via eikonalization corresponds to the lowest $Q \bar{Q}$ Fock component, $|Q \bar{Q}\rangle$. It does not include any correction for gluon shadowing, but rather corresponds to shadowing of sea quarks in nuclei. Although $\sigma_{\text {dip }}$ includes all possible effects of gluon radiation, the eikonal assumes that none of the radiated gluons take part in multiple interactions in the nucleus. The leading order correction corresponding to gluon shadowing comes from the eikonalization of the next Fock component $|Q \bar{Q} G\rangle$. Explicitly, in the large coherence length limit $\ell_{c} \rightarrow \infty$ the general formula for the $\gamma A$ cross section is given by

$$
\begin{aligned}
\frac{d^{2} \sigma_{\gamma A}}{d^{2} b}= & 2 \int d z \int d^{2} r\left|\psi_{q \bar{q}}\right|^{2}\left[1-\exp \left(-\frac{1}{2} \sigma_{q \bar{q}} T_{A}(b)\right)\right] \\
& +2 \int d z \int \frac{d z_{G}}{z_{G}} \int d^{2} r_{1} \int d^{2} r_{2}\left|\psi_{q \bar{q} G}\right|^{2} \\
& \times\left[1-\exp \left(-\frac{1}{2} \sigma_{q \bar{q} G} T_{A}(b)\right)\right]
\end{aligned}
$$

where $\psi_{q \bar{q} G}$ is the wave function for the gluonic component and the cross section for the three-body system $\sigma_{q \bar{q} G}$ can be expressed in terms of the dipole cross section, $\sigma_{\text {dip }}$. The complete calculation has been done in Ref. [15], and it was shown that the $|Q \bar{Q} G\rangle$ contribution can be absorbed in a factor $R_{G}\left(x, Q^{2}, b\right)$ multiplying the original dipole cross section. The evaluation of the gluon shadowing contribution in [15] is not trivial and somewhat complex for practical use.

In Ref. [7], the gluon shadowing correction was introduced and the model sensitivity was analyzed. It was found that a strong model dependence on predictions existed. For our purpose here, we will use the simple parametrization of $R_{G}(x, b)$ given by HIJING 2.0 [16] (with $s_{g}=0.17$ ), which accounts for the impact parameter dependence of the nuclear gluon ratio. As a cross-check, in Fig. 1 we show the results using $R_{G}=1$ (dot-dashed curve) and the impact parameter gluon ratio (long-dashed curve). The last option is good enough for the remaining discussions. Despite the gluon shadowing being an issue, the photon-proton contribution dominates as the photon flux emitted by the nucleus is enhanced compared to the one coming from the proton and the corresponding nuclear suppression of $\sigma_{\gamma A \rightarrow V A}$. For lead-proton collisions at $5.02 \mathrm{TeV}$ and rapidity $y=-3$ the quarkonium production on the proton (low-energy photons from the nucleus) probes Bjorken variable values $x_{p} \simeq 10^{-2}$ whereas the production on the nucleus (high-energy photons from proton) probes values $x_{A} \simeq 10^{-5}$. In Fig. 2 we present the results for the rapidity distribution of the $\psi(1 S)$ state in $\mathrm{Pb}+p$ collisions at $\sqrt{s_{p A}}=5.02 \mathrm{TeV}$. The dot-dashed curve is obtained using $R_{G}=1$ and the long-dashed one is for the $R_{G}(x, b)$ from HIJING 2.0. As expected the gluon shadowing effect has a 


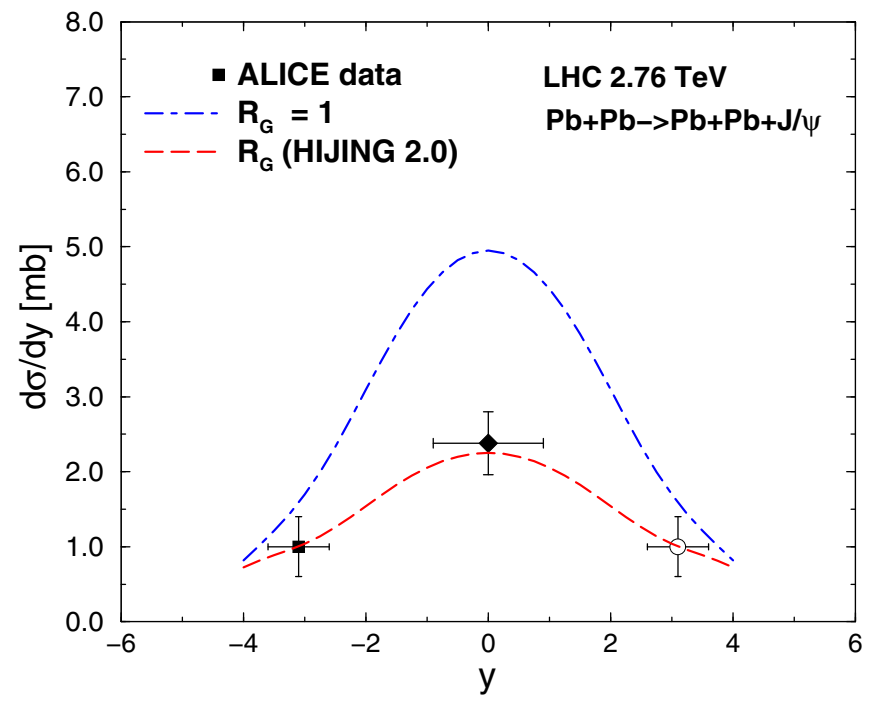

FIG. 1. (Color online) Rapidity distribution of $J / \psi$ photoproduction in $\mathrm{PbPb}$ collisions at $2.76 \mathrm{TeV}$ compared to ALICE data $[13,14]$. The dot-dashed curve represents the calculation using $R_{G}=1$ and the long-dashed one is for the HIJING 2.0 nuclear ratio.

small impact in the $p A$ case as the photon-proton interaction is a minor contribution and it is relevant in the negative rapidity region (small $x_{A}$ ). We have checked that the rapidity distribution is suppressed by a factor 0.72 at $y=-5$ to 0.94 at $y=0$. In addition, in Fig. 3 we present the results for the excited state $\psi(2 S)$, using the same notation for the figure labels. The suppression is similar to the $J / \psi$ case, being a factor 0.62 at $y=-5$ as expected due the node effects (more shadowing compared to the fundamental state).

Finally, in Fig. 4 the investigation for the $\Upsilon(1 S)$ state is done. Clearly, the suppression in negative rapidities is stronger than for charmonia, mostly for $y<-2$. This is due to the fact

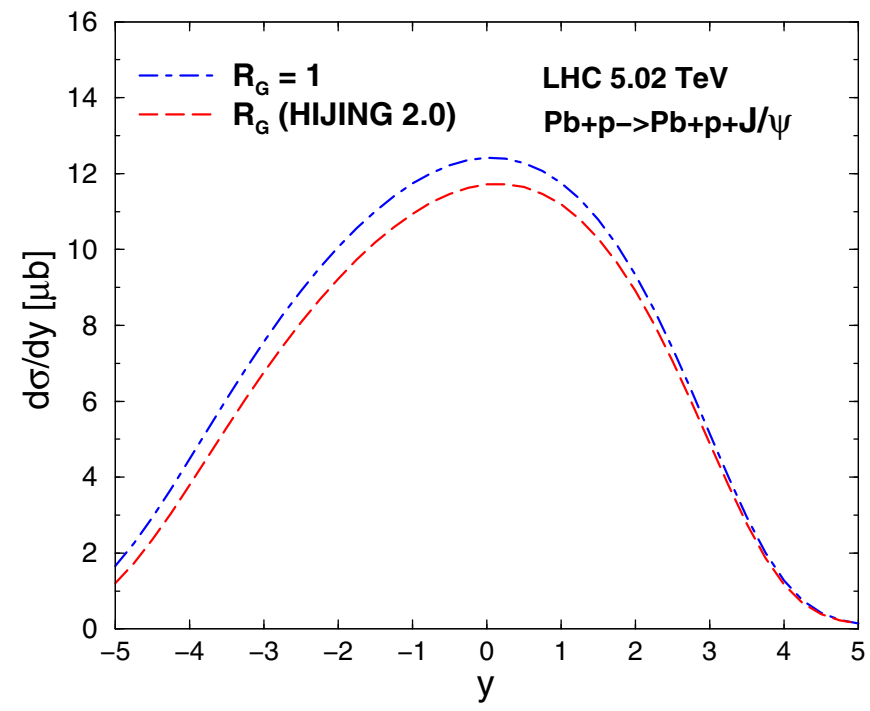

FIG. 2. (Color online) Rapidity distribution of $\psi(1 S)$ production in $p A$ collisions at $5.02 \mathrm{TeV}$ (curves are the same notation as in Fig. 1).

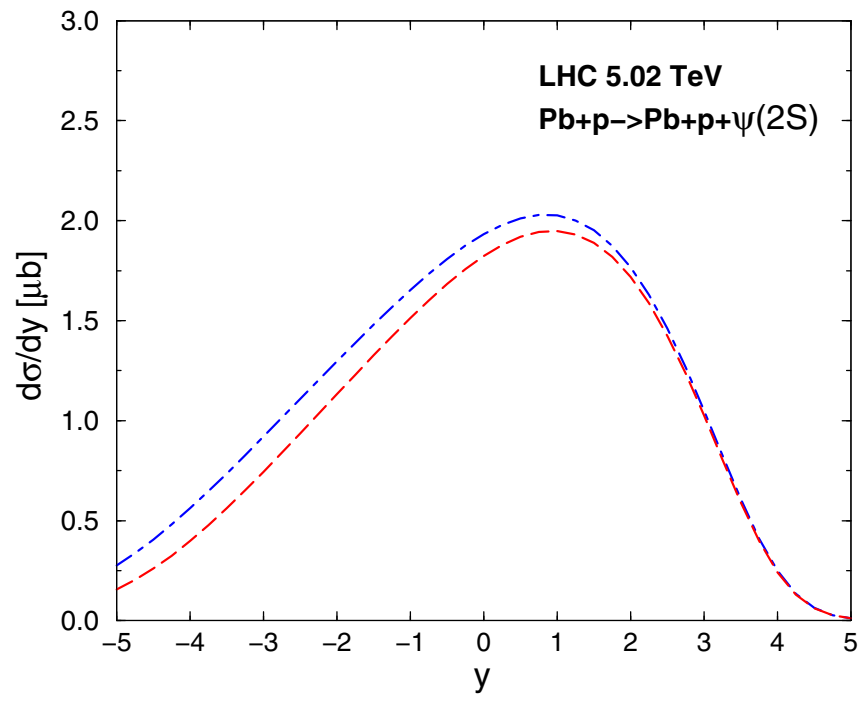

FIG. 3. (Color online) Rapidity distribution of $\psi(2 S)$ production in $p A$ collisions at $5.02 \mathrm{TeV}$ (curves are the same notation as in Fig. 1).

that the photon-nucleus interaction contributes more in the $\Upsilon$ case compared to charmonia. Namely, the eikonal giving the multiple scattering of color dipoles is less suppressed compared to $J / \psi$ production. That is directly related to the higher $x_{A}$ probed in $\Upsilon$ production. For instance, at $y=-3$ one gets $x_{A} \simeq 10^{-4}$ that can be compared to values $x_{A} \simeq 10^{-5}$ for charmonia at the same rapidity value.

The predictions for the higher energy $p A$ collisions are presented in Fig. 5, taking the designed energy of $\sqrt{s_{p A}}=$ $8.8 \mathrm{TeV}$. In both plots, we are using $R_{G}(x, b)$ from HIJING 2.0. In Fig. 5(a) we analyze the charmonia production. The solid curve represents the result for $J / \psi$ production, whereas the prediction for $\psi(2 S)$ is given by the dashed curve. The ratio $\psi(2 S) / \psi(1 S)$ follows the original trend at $5.02 \mathrm{TeV}$ and the cross section is higher by a factor 1.3. In Fig. 5(b), the

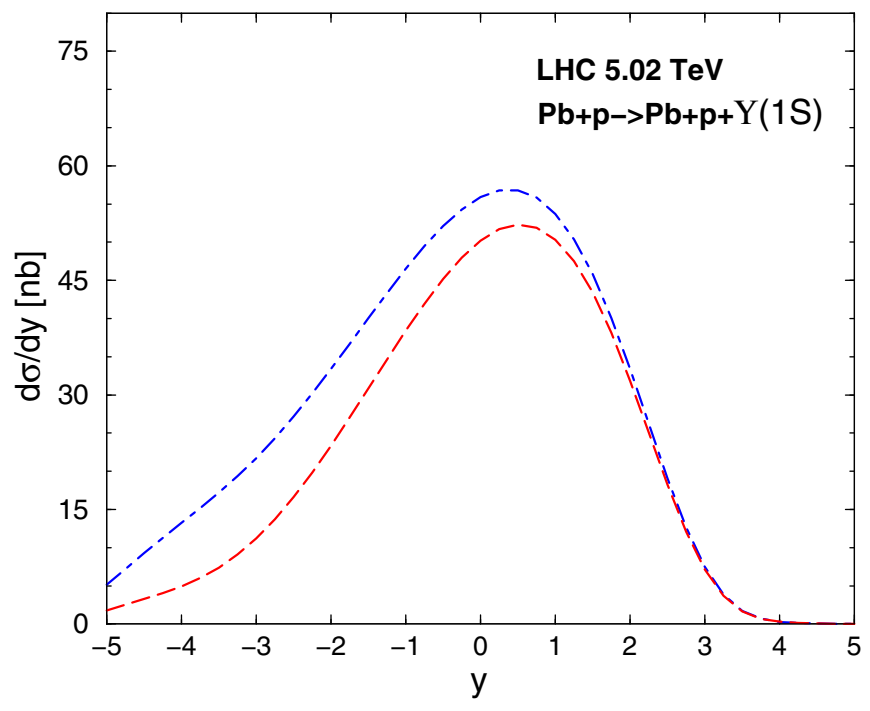

FIG. 4. (Color online) Rapidity distribution of $\Upsilon$ production in $p A$ collisions at $5.02 \mathrm{TeV}$ (curves are the same notation as in Fig. 1). 


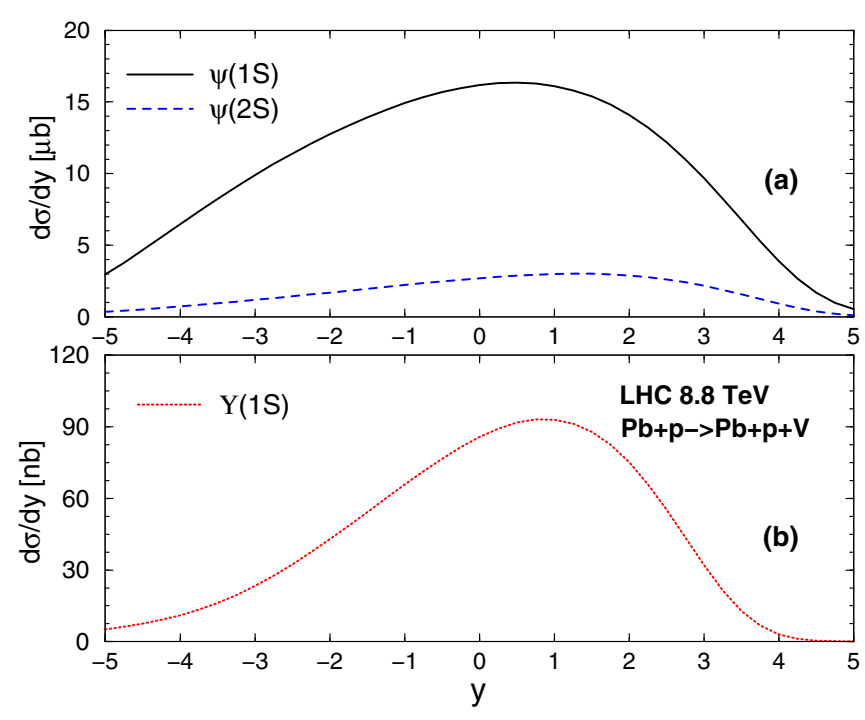

FIG. 5. (Color online) (a) The rapidity distribution for $\psi(1 S)$ (solid line) and $\psi(2 S)$ production (dashed line) at energy $\sqrt{s_{p A}}=$ $8.8 \mathrm{TeV}$. (b) The rapidity distribution for $\Upsilon(1 S)$ production at energy $\sqrt{s_{p A}}=8.8 \mathrm{TeV}$.

prediction for $\Upsilon$ is done. The enhancement in cross section normalization is now a factor 1.5 .

Let us now compare the present results to other approaches. In Ref. [17] the $J / \psi$ production in $p A$ has been computed in $5.02 \mathrm{TeV}$ using the LO pQCD calculations. The prediction for the rapidity distribution for $\mathrm{Pb}+p \rightarrow \mathrm{Pb}+p+J / \psi$ is qualitatively similar to ours. The main difference is in the very forward negative rapidities, where the distribution is smaller than in [17]. The reason is the distinct way to parametrize the threshold $x_{g} \rightarrow 1$ effect in the photon-proton cross section. A similar LO pQCD calculation has also been done in Ref. [18] for the $J / \psi$ and $\Upsilon$ production. Our predictions are similar to results using the EKS08 nuclear PDF in [18], despite not having a second peak for $\Upsilon$. The reason should be a stronger suppression for heavier mesons in our case (the HIJING 2.0 gluon ratio gives a very strong shadowing effect). When compared to approaches using the color dipole framework, the additional information here is the introduction of the gluon shadowing correction which is not so intense as in the $\mathrm{PbPb}$ case at the LHC energies. In Ref. [19], the production of $J / \psi$ has been computed in $p A$ collisions and our results are similar except in the large negative rapidity region. This is due to our $x_{p}$-threshold factor imposed on the dipole cross section. Compared to the previous $p A$ calculation in the color dipole framework [20], the current study introduces the additional contribution from the photon-nucleus interaction.

Finally, let us comment on the theoretical uncertainties associated to the calculations. As pointed out in Ref. [17], the photon flux for protons introduces some uncertainty mostly at large rapidities whereas deviations in modeling the flux for the nucleus is considerably smaller. Concerning the color dipole framework considered to compute the photon level cross sections, the main uncertainties come from the model for the meson wave function and on the choice for the dipole-proton cross section. The uncertainty on modeling the wave function gives an uncertainty of order 13\% (for fixed dipole cross section), whereas one has $5 \%$ uncertainty on modeling the dipole cross section in the $p A$ case. We would have a considerable uncertainty on the nuclear shadowing factor $R_{G}\left(x, Q^{2}, b\right)$, which is very important in the negative rapidity region. We have constrained it by describing the $J / \psi$ cross section in $\mathrm{PbPb}$ collisions measured by ALICE.

\section{SUMMARY}

An investigation was done on the coherent photoproduction of charmonia and $\Upsilon$ in the proton-nucleus collisions in the LHC energies. It included both contributions of photon-proton and proton-nucleus interactions. Predictions for the rapidity distributions are presented using the color dipole formalism and including saturation effects that are expected to be relevant at high energies. It was found that the photon-nucleus contribution is not so important for the charmonium production but relevant for the $\Upsilon$ case. The calculation is consistent with other approaches, such as the LO pQCD formalism and previous color dipole calculations. The gluon shadowing effect is considered and the impact is smaller than for the $\mathrm{PbPb}$ case. Predictions were made for the next run of the LHC considering proton-lead collisions and the main features present at $5.05 \mathrm{TeV}$ are still present.

\section{ACKNOWLEDGMENTS}

This work was partially financed by the Brazilian funding agency CNPq and by the French-Brazilian scientific cooperation project CAPES-COFECUB 744/12. M.V.T.M. thanks the kind hospitality at IPhT CEA Saclay (France), where this work was accomplished.
[1] N. N. Nikolaev and B. G. Zakharov, Phys. Lett. B 332, 184 (1994); Z. Physik C 64, 631 (1994).

[2] J. Nemchik, N. N. Nikolaev, E. Predazzi, and B. G. Zakharov, Phys. Lett. B 374, 199 (1996).

[3] F. Gelis, E. Iancu, J. Jalilian-Marian, and R. Venugopalan, Ann. Rev. Nucl. Part. Sci. 60, 463 (2010); H. Weigert, Prog. Part. Nucl. Phys. 55, 461 (2005); J. Jalilian-Marian and Y. V. Kovchegov, ibid. 56, 104 (2006).

[4] G. Baur, K. Hencken, D. Trautmann, S. Sadovsky, and Y. Kharlov, Phys. Rep. 364, 359 (2002); C. A. Bertulani, S. R. Klein, and J. Nystrand, Ann. Rev. Nucl. Part. Sci. 55, 271 (2005).
[5] S. R. Klein and J. Nystrand, Phys. Rev. C 60, 014903 (1999).

[6] C. Adloff et al. (H1 Collaboration), Phys. Lett. B 541, 251 (2002).

[7] M. B. Gay Ducati, M. T. Griep, and M. V. T. Machado, Phys. Rev. D 88, 017504 (2013); Phys. Rev. C 88, 014910 (2013).

[8] B. Z. Kopeliovich and B. G. Zakharov, Phys. Rev. D 44, 3466 (1991).

[9] Y. P. Ivanov, B. Z. Kopeliovich, A. V. Tarasov, and J. Hufner, Phys. Rev. C 66, 024903 (2002). 
[10] J. R. Forshaw, R. Sandapen, and G. Shaw, J. High Energy Phys. 11 (2006) 025; B. E. Cox, J. R. Forshaw, and R. Sandapen, ibid. 06 (2009) 034.

[11] E. Iancu, K. Itakura, and S. Munier, Phys. Lett. B 590, 199 (2004).

[12] A. H. Rezaeian and I. Schmidt, Phys. Rev. D 88, 074016 (2013).

[13] B. Abelev et al. (ALICE Collaboration), Phys. Lett. B 718, 1273 (2013).

[14] E. Abbas et al. (ALICE Collaboration), Eur. Phys. J. C 73, 2617 (2013).
[15] B. Kopeliovich, A. Schäfer, and A. Tarasov, Phys. Rev. D 62 , 054022 (2000).

[16] W.-T. Deng, X.-N. Wang, and R. Xu, Phys. Rev. C 83, 014915 (2011).

[17] V. Guzey and M. Zhalov, arXiv:1307.6689 [hep-ph].

[18] A. Adeluyi and T. Nguyen, Phys. Rev. C 87, 027901 (2013).

[19] T. Lappi and H. Mantysaari, Phys. Rev. C 87, 032201(R) (2013).

[20] V. P. Gonçalves and M. V. T. Machado, Phys. Rev. C 73, 044902 (2006) 